\title{
9. Warum sagen die Aktivist*innen Ja zum queeren Wir?
}

Im diskursanalytischen Teil der Arbeit wurde zunächst herausgearbeitet, auf welche Art und Weise die fünf im Zentrum der vorliegenden Untersuchung stehenden queeren Projekte ein kollektives queeres Wir herstellen. Im Folgenden soll es nun darum gehen, welche der in den queeren Diskursen hergestellten Bedeutungsanordnungen von den Akteur*innen erkannt werden und wie sie sich warum dazu ins Verhältnis setzen. Hierzu wird im ersten Abschnitt herausgearbeitet, welche Rolle der Begriff »queer « als kollektive und individuelle Verortung für die Aktivist*innen spielt. Im zweiten Abschnitt werden von den im diskursanalytischen Teil der vorliegenden Arbeit untersuchten, diskursiv hergestellten Bedeutungsanordnungen diejenigen hervorgehoben, auf die die Aktivist*innen Bezug nehmen und die aus ihrer Perspektive relevant sind. Dem folgen im dritten Abschnitt weitere Bedingungs-/Bedeutungs-/Begründungsanordnungen, die aus der Perspektive der Aktivist*innen von Bedeutung sind. Hierbei handelt es sich vor allem um Widersprüche zwischen den strukturellen Bedingungen des politischen Aktivismus und den persönlichen Bedürfnissen der Akteur*innen, die auch auf bestimmte queere Bedeutungsanordnungen zurückgehen. Es geht aber auch um das je eigene Leben der Akteur*innen, das in den Begründungen für/gegen den Aktivismus ebenfalls eine Rolle spielt.

\subsection{We are queer - was heißt das eigentlich?}

Im Interviewleitfaden war die Bedeutung des Begriffs »queer « für die Akteur*innen explizit Thema. Im Sinne der Bedingungs-Bedeutungs-Begründungsanalyse ging es um die Frage, inwiefern das jeweilige Projekt als »queer« verstanden wird (subjektive Bedeutungsebene), wie sich die Aktivist*innen zu diesem »queer « positionieren (Positionierungen zum queeren Wir) und warum sie sich auf eine bestimmte Art dazu positionieren (Begründungsebene).

Die Analyse der subjektiven Bedeutungsebene zeigt, dass die Aktvist"innen dem Begriff "queer « unterschiedliche Bedeutungen zuschreiben: Die interviewten Personen verstehen die jeweiligen queeren Einrichtungen, von denen sie Teil sind, auf drei verschiedene Arten als »queer«: erstens, insofern sie queer handeln (Villa, Lambda BB); 
zweitens, insofern sie eine queere Haltung teilen (Milchjugend, Lambda BB); und drittens, insofern queer als Überbegriff beschreibt, wer Teil von dem Projekt ist (TrIQ, LesMigraS). ${ }^{1}$

Die Villa wird beispielsweise als Ort verstanden, der queer handelt. Konkret heißt das, dass die Villa als ein offener Raum beschrieben wird, an dem ein vielfältiges $\mathrm{Ne}$ beneinander möglich ist:

»Sie ist wahnsinnig >queer im Sinne, dass sie einfach ein offener Raum ist und Leuten Ressourcen und Räume zur Verfügung stellt, um dort zu arbeiten. Ob das jetzt alte Männer sind, die sich jetzt zu Religion oder Religionsthemen treffen, oder ob das jetzt junge Schwarze Trans"-Aktivist*innen sind, ist ziemlich nebensächlich. [...] So. Aber wir haben trotzdem Platz, auch wenn sie sich nicht als >queer <identifizieren, und ich glaub, das ist >queer< - so ganz billig. >Queer ist kein Label. >Queer ist tun.«

Die Aktivist"innen der Milchjugend hingegen verstehen "queer als eine gemeinsam geteilte Haltung, die die Einrichtung zusammenhält: »Aber wir bringen niemanden bei, dass sie sich als falsch ${ }^{2}$ verstehen müssen. [...] Es ist sicher kein Identitätsbegriff. Es kann diese Funktion nicht ersetzen und sie ist trotzdem nötig, aber es hilft, dass alle dabei sein können, die quasi eine Haltung teilen. «Queer als Haltung zu verstehen, bedeutet aus der Perspektive der Aktivist"innen, eine kritische Beziehung zur Norm einzunehmen. Kritik an der Norm wird allerdings einmal als reine Negation verstanden: »[e]in Begriff als direktes Gegenkonzept zur Norm. Der Normen generell ablehnt « (Lambda $B B$ ), einmal als Politik des Stolzes: »Wir sagen sja<, wir wollen nicht in die Norm und so sein wie alle anderen, sondern wir sind eigentlich//wir fühlen uns wohl, so wie wir sind« (Milchjugend). Queer wird aber auch als Überbegriff, der die Personen beschreibt, die Teil des Projektes sind, verstanden: »Ja, ich glaube aus meiner Erfahrung heraus, dass es viele queere Leute gibt, die Teil davon sind «(TrIQ). Die Art, wie die Menschen aus dem Projekt sich selbst bezeichnen oder verorten, bestimmt diesem Verständnis zufolge das queere Wir.

Die subjektiven Bedeutungen, die die Aktivist*innen dem queeren Wir ihrer Gemeinschaften zuordnen, knüpfen mitunter an Aspekte des diskursiv hergestellten Wir der jeweiligen Projekte an. Die Villa konstituiert sich, ähnlich wie es in den subjektiven Bedeutungen gefasst wurde, auch in den analysierten Diskursen der vorliegenden Arbeit als Einrichtung, die insofern »queer« ist, als queer gehandelt wird. Die Villa fordert beispielsweise nicht einfach nur mehr Räume für Mehrfachmarginalisierte auf der Pride, das Projekt schafft selbst diese Räume, indem es mit einem eigenen »queer-feministischen, migrantischen und antirassistischen Truck« auf der Pride erscheint. Auch das diskursiv hergestellte Wir der Milchjugend knüpft an der subjektiven Bedeutung von »queer« als gemeinsam geteilte stolze Haltung an. Bei TrIQ und LesMigraS sind die Bezüge zwischen den subjektiven und diskursiv hergestellten Bedeutungen insofern gegeben, als die Personen, die Teil der jeweiligen Einrichtungen sind, eine zentrale Rolle

Dem Begriff »queer« wird in Bezug auf die Projekte mal mehr und mal weniger Bedeutung zugeschrieben. Die interviewte Person von LesMigraS betont beispielsweise eher das Intersektionale und weniger das Queere des Vereins.

2 Die Milchjugend bezeichnet sich selbst als »falschsexuell« und versteht diesen Begriff als eine Übersetzung von »queer «. 
für die Konstitution der jeweiligen Gemeinschaft spielen. LesMigraS stellt beispielsweise die Interessen von Mehrfachmarginalisierten in den Mittelpunkt der eigenen Arbeit und damit auch dessen, »was die Einrichtung ist«.

Auch die Art und Weise wie sich die einzelnen Aktivist"innen zum queeren Wir jeweils positionieren fällt unterschiedlich aus: Aus der Perspektive der Aktivist"innen nimmt das "Queer« ihrer Einrichtung verschiedene Bedeutungen an. Wie aber setzen sich die Akteur*innen in ein Verhältnis zu dem so verstandenen queeren Wir? Wird es angenommen oder abgelehnt? Und wie stehen individuelle und kollektive Selbstverortung in Beziehung zueinander? Die Aktivist*innen verorten sich zunächst unabhängig davon, auf welche Art und Weise das jeweilige Projekt als »queer « verstanden wird, selbst als »queer«. Manche lehnen den Begriff als Selbstbezeichnung ab: »Queer als Selbstverortung finde ich schwierig, weil man da an sehr viele Grenzen gerät, aber das ist jetzt meine persönliche Sicht und ich glaub, für viele Leute funktioniert der Begriff sehr gut, weil er einfach den Vorteil hat, dass er unsere Identitäten negiert.«(IP3) Andere hingegen verstehen sich selbst als »queer«: »Also ich persönlich würde mich da durchaus auch selbst verorten. Also ich würd' mich auch durchaus als irgendwie >queer bezeichnen. Ich finde, es ist auch >ne Selbstbezeichnung. «(IP4) Es wird aber auch davon gesprochen, dass sich der persönliche Bezug zu »queer « als Selbstbezeichnung verändert habe: »Das heißt, >queer < ist ein Begriff, den ich ursprünglich dankbar angenommen habe, der aber im Laufe der Jahre ein bisschen von seinem Charme verloren hat.«(IP6)

Während es für die Selbstverortung als "queer« keine Rolle spielt, ob das "Queer« des eigenen Projektes als Haltung, Handlung oder Überbegriff verstanden wird, spielt dieses Verständnis sehr wohl eine Rolle, wenn es darum geht, wie die Akteur*innen sich $\mathrm{zu}$ ihrer queeren Gemeinschaft positionieren. Diejenigen, die das "Queer« ihrer Projekte als gemeinsame Haltung oder Handlung verstehen, verorten sich alle - unabhängig davon, welche Rolle »queer« als individuelle Selbstverortung für sie spielt - im kollektiven queeren Wir ihrer Einrichtungen. Manche lehnen eine queere Selbstbezeichnung ab, bejahen aber ein kollektives queeres Wir: »Aber ich glaub, von meinem Denken her denke ich, dass ich >queer bin, oder ich hoffe, dass ich >queer < bin, aber als Selbstbezeichnung nutzt es mir nichts.« (IP3) Eine andere Person nimmt "queer« sowohl als individuelle als auch kollektive Bezeichnung an: »Also der Begriff kann beides sehr gut sein.«(IP4) Im Unterschied dazu sagen jene Aktivist*innen, die queer als Überbegriff verstehen, Nein zu einem kollektiven queeren Wir. Ein Aktivist* versteht sich beispielsweise selbst als »queer«, lehnt die Bezeichnung aber als Überbegriff ab: »Und ich bezeichne mich selber auch als >queer und gleichzeitig finde ich ihn so als Überbegriff sehr tricky.« (IP5) Aus der Perspektive der ersten Person ist keine individuelle Identifizierung mit dem Begriff »queer" notwendig, um ihn als kollektiven Begriff anzunehmen. Es macht allerdings einen Unterschied, wie die Aktivist"innen das "Queer« der eigenen Gemeinschaft verstehen. "Queer« verstanden als Handlung oder Haltung ist eine Gemeinschaftsbezeichnung, die aus der Perspektive der Aktivist"innen annehmbar ist, während »queer« verstanden als Überbegriff eher abgelehnt wird.

Nachdem nun aufgezeigt wurde, welche Bedeutungen die Aktivist*innen in Bezug auf das kollektive queere Wir ihrer Projekte erkennen und wie sie sich konkret dazu po- 
sitionieren, soll nun im nächsten Schritt die Begründungsebene dargestellt werden: Warum nehmen die Aktivist"innen queer als Haltung oder Handlung eher an, als queer als Überbegriff? "Queer wird von den Aktivist*innen als kollektive Bezeichnung angenommen, weil es ein Mehr als Identität verspricht: »[w]ährend ich >queer mehr so als einen politischen Begriff sehe und auch als einen Begriff, der sehr, sehr viel mehr aussagt als zum Beispiel Identität« (IP4). Dabei werden drei Aspekte hervorgehoben, die das Mehr als Identität möglich machen. "Queer« ist aus der Perspektive der Akteur"innen insofern mehr als Identität, als der Begriff erstens Bündnisse möglich macht, zweitens subversiv ist und drittens ein geeignetes Repräsentationsmittel darstellt. Die Bezeichnung "queer« ist aus der Perspektive der Aktivist"innen erstens von Bedeutung, weil "queer« Bündnisse zwischen verschiedenen Personengruppen jenseits von eindeutigen Identitäten möglich macht:

»Also es ist ein Begriff, der irgendwie eine gewisse Einigkeit schafft. Weil es ein Begriff ist, der sehr, sehr viele Sachen unter einen Hut bringt und halt so den gemeinsamen Kampf mit zum Beispiel cis-schwulen Männern, mit denen ich halt sonst nicht so viel zu tun habe in allen Bereichen meines Lebens, der aber selbst mit solchen Leuten eine gemeinsame Idee irgendwo schafft.«(IP4)

Die Aktivist*innen wollen aber zweitens auch eine queere Gemeinschaft, weil »queer « die Möglichkeit subversiver Politiken eröffnet: »[D]u kannst Mehrheiten schaffen, $d u$ kannst auch provozieren wahnsinnig gut über den Begriff, du kannst irritieren, $d u$ kannst subvertieren, du kannst spielen, du kannst dich ausprobieren, du kannst dich austoben.«(IP3) Das kollektive queere Wir wird drittens bejaht, weil »queer« ein geeignetes Repräsentationsmittel darstellt: »Also es ist mehr so ein Mittel für uns auch gegen außen, für die Kommunikation.«(IP2) Die Personen, die sich selbst in ein bejahendes Verhältnis zum kollektiven queeren Wir ihrer Projekte setzten, erhoffen sich von dem Begriff zu gewinnen, was er theoretisch wie bewegungsgeschichtlich verspricht: Bündnisse, Repräsentation, Subversion - mehr als Identität.

"Queer« wird umgekehrt von den Aktivist*innen als ein- und ausschließender Begriff abgelehnt. "Queer « wird abgelehnt, wenn der Begriff erstens als Selbstbezeichnung zur Bedingung wird, zweitens synonym für eine Gruppe steht, drittens bestimmte Gruppen per se ausschließt. "Queer« wird als ein kollektiver Begriff von den Aktivist"innen verneint, wenn eine Selbstverortung als "queer« die Bedingung dafür wird, Teil der so bezeichneten Gemeinschaft sein zu können: »Jetzt vor allem spezifisch auf Inter*Kontexte, genau da würde ich da unter Umständen vorsichtig sein, inwiefern das Queer oft dann schnell auch im Vordergrund steht und dann natürlich die Frage ist, was ist jetzt mit den Personen, die zum Beispiel inter* sind und sich nicht als queer verstehen, haben die da auch Platz?« Sich selbst als »queer « zu verorten, soll aus der Perspektive der Aktivist*innen keine Bedingung für das kollektive queere Wir sein. Für die Akteur*innen ist es aber auch wichtig, dass "queer « nicht insofern ein ein- und ausschließender Begriff ist, als er nur synonym für eine Identitäts-Kategorie verwendet wird:

»In den Jahren, die danach kamen, wurde es aber leider zum neuen Modebegriff und synonym für schwul, wodurch es komplett an Bedeutung verloren hat. Sprich [...] die Leute denken, squeer ist einfach nur ein Wort für schwul. Und das hat dem ganzen 
so ein bisschen den Saft rausgezogen, weil da das Infragestellen von Geschlecht vollkommen fehlt, und das finde ich problematisch, denn es ist nicht einfach synonym für schwul.«(IP6)

Auf der anderen Seite wird aber auch ein Verständnis von »queer« abgelehnt, das bestimmte Gruppen - vor allem Schwule - per se ausschließt:

»Dass schwule Männer, die sich als >queer bezeichnen, cultural appropriation betreiben. Wo ich mir denke, so naa, das hat der Begriff so nie in sich gehabt! [...] >Queer < ist nicht Schwarz und nicht weiß und nicht schwul und nicht lesbisch. Es ist halt einfach ein Konstrukt, ein temporärer Begriff, der einfach sagt, Identitätspolitiken werden uns nicht retten.«(IP4)

"Queer « wird also als ein Begriff abgelehnt, der ausschließend ist, weil er zur Bedingung wird oder weil er eine eindeutige Identitäts-Kategorie beschreibt, aber auch als Begriff, der bestimmten Gruppen per se verwehrt wird.

Gemeinsam ist allen Aktivist*innen, dass sie bestimmte als identitätspolitisch geltende Formen von »queer ablehnen beziehungsweise Ja sagen zu bestimmten Möglichkeiten von »queer« jenseits von einfachen Identitätspolitiken. Die subjektiven Gründe für oder gegen ein Ja zum queeren kollektiven Wir sind eng verknüpft mit den bewegungsgeschichtlichen und theoretischen Versprechen von »queer«. "Queer« wird als eine kollektive Bezeichnung angenommen, die statt ein- und ausschließenden Identitätspolitiken Bündnisse und subversive Politiken möglich macht. Wie sich die einzelnen Aktivist"innen in ein Verhältnis zu dem »Queer« ihrer Gemeinschaft setzen, hängt grundlegend damit zusammen, inwiefern dem "Queer « ihrer Gemeinschaft ein Mehr als Identität zugestanden wird. Es scheint aus der Perspektive der ersten Person von Bedeutung zu sein, sich um einen Ort zu versammeln, der nicht ein- und ausschließt und der ein Jenseits von einfachen Identitätspolitiken in Form von Bündnissen, Subversion und strategischer Repräsentation möglich macht.

\subsection{Mehrfachdiskriminierung - CSD - Coming-out}

Welche anderen Bedeutungs- und Begründungsdimensionen spielen eine Rolle in der Art, wie die einzelnen Personen sich zu dem kollektiven Wir ihrer Projekte ins Verhältnis setzten? Die Aktivist*innen nehmen nicht nur Bezug auf den Begriff »queer«, sondern auch - in diesem Fall ohne explizite Frage danach - auf die in den Öffentlichkeiten der Projekte verhandelten zentralen Themen: Mehrfachdiskriminierung, Pride und Coming-out. Während die Personen aus der Villa und von LesMigraS Mehrfachdiskriminierung, die auch in ihren Projekten jeweils zentral ist, thematisieren, beziehen sich die Aktivist*innen der beiden queeren Jugendgruppen auf die Pride und das Coming-out - also auch hier die zentralen Themen der jeweiligen projekteigenen Öffentlichkeiten. Die Person von TrIQ hingegen greift nicht explizit auf einen der drei zentralen 
Diskursstränge zurück. Hier wird stattdessen die Bedeutung der Einrichtung als Austauschraum betont. ${ }^{3}$

\section{Mehrfachdiskriminierung}

An die diskursiven Aushandlungen von LesMigraS, in denen die Thematisierung von Mehrfachdiskriminierung eine zentrale Rolle spielt, anknüpfend, betont auch die bei LesMigraS arbeitende interviewte Person die Bedeutung des intersektionalen Ansatzes der Einrichtung für das eigene Engagement. Auf der subjektiven Bedeutungsebene stellt sich auch hier zunächst die Frage, welches intersektionale Wir aus der Perspektive der Aktivist*innen erkannt wird. Mehrfachdiskriminierung wird von der interviewten Person als eine alltägliche und existenzielle Erfahrung verstanden. Das Alltägliche wird in Abgrenzung zu einem Verständnis von Intersektionalität »als theoretisches Steckenpferd « betont. Demgegenüber wird Mehrfachdiskriminierung als »Lebensrealität« verstanden, mit der die interviewte Person »tagtäglich « zu tun hat. Diese subjektive Bedeutung knüpft an die diskursiven Aushandlungen von LesMigraS an. Auch wenn dort selten eine explizite Abgrenzung zu einem akademischen Intersektionalitätsbegriff stattfindet, geht die politische Arbeit der Einrichtung konsequent von den Erfahrungen der von Gewalt und Mehrfachdiskriminierung betroffenen Personen aus. Anders als in den diskursiven Aushandlungsprozessen von LesMigraS, wird aus der Perspektive der ersten Person auch das Existenzielle von Mehrfachdiskriminierungserfahrungen betont. Eine intersektionale Gemeinschaft ist ein Wir, das gemeinsam gegen prekäre Lebensverhältnisse kämpft:

»Und ich rede tatsächlich von Überleben. Ich kenne sehr viele Leute, die tatsächlich kein Dach über dem Kopf haben. Ich kenne so viele Leute, die haben nicht genug zu essen [...]. Ich kenne Leute, die haben einen Master und die haben tatsächlich, wir reden hier gerade mal von WG-Zimmern und die sind nicht mal drin. Genauso wie ich Leute kenne, die total intelligent, aber illegalisiert hier sind.«

Es wird ein intersektionales Wir bejaht, das sich ausgehend von alltäglichen Mehrfachdiskriminierungserfahrungen für ein Überleben einsetzt. Dabei werden zwei Gründe genannt, warum ein solches intersektionales Engagement aus der Perspektive der ersten Person relevant ist: zum einen aus einer strukturellen Notwendigkeit, zum anderen aus einer affektiven Verbundenheit heraus. Eine Beteiligung am intersektionalen Aktivismus wird als bedeutungsvoll erachtet, weil andere Institutionen auf die Notwendigkeit solcher alltäglicher existenzieller Erfahrungen nicht geantwortet hätten. Es gebe keine Fördermöglichkeiten an der Schnittstelle von Diskriminierung und Gesundheit, Europa tue nichts, die Gender Studies würden nicht verstehen und die Psychotherapeut"innen würden sich nicht auskennen:

»Im Gegenteil, es sind extrem intelligente Leute, die nicht verstehen, warum so viele Trans*-Menschen of Colour sterben, jung sterben, früh sterben. Und scheinbar ist da so >ne fast schon kindliche Vorstellung von - naja - irgendwas müssen die ja falsch 
gemacht haben. Nein! Nein! Wenn du in einem System lebst, in dem du eh schon wegen der Hautfarbe schlecht behandelt wirst, und dann kommt der Trans*-Faktor hinzu. Und das ist kein additiver Effekt. Sondern das führt dazu, dass das Stigma und die Cefahrenquellen implizit unendlich werden. Dann wird auf einmal deutlicher, warum so viele von uns sterben. [...] Und wenn das was ist, was akademisierte hoch intelligente Leute nicht verstehen, dann weiß ich gut, dann werde ich wenigstens ein paar Jahre meines Lebens einer unterbezahlten NCO widmen, um einen kleinen Teil beizutragen, dass ein paar Leute weniger sterben.«

Ein Grund, sich für ein intersektionales politisches Engagement $\mathrm{zu}$ entscheiden, ist die Notwendigkeit, auf alltägliche existenzielle Mehrfachdiskriminierungserfahrungen zu antworten, weil sonst niemand darauf antwortet. Der zweite Grund, der für die Entscheidung für ein intersektionales Wir eine Rolle spielt, ist eine persönliche affektive Verbundenheit mit denjenigen, die alltäglich existenzielle Mehrfachdiskriminierungserfahrungen machen. Die interviewte Person berichtet davon, viele Menschen zu kennen, die an der Schnittstelle von verschiedenen Diskriminierungen um ein Überleben kämpfen: »Es ist wichtig geworden unter anderem, weil ich so viele Geschichten gehört habe von Leuten, die mir am Herzen lagen, die ums Überleben kämpfen. [...] Und das sind Leute, mit denen du feierst, mit denen du ins Bett gehst, die du lieb hast, weißt du, die dir am Herzen liegen.«

Auch wenn Intersektionalität der Begriff ist, mit dem dieses Engagement bezeichnet wird, knüpfen die subjektiven Bedeutungen und Begründungen in mehrfacher Hinsicht an das bewegungsgeschichtliche "Queer« an. In der Aids-Krise formierte sich "queer« als gemeinsamer Kampf gegen den Tod. Auch dort war »queer« eine Antwort auf den Umstand, dass die amerikanische Politik nichts gegen das Sterben unternahm (Reagan schwieg acht Jahre zu Aids). ${ }^{4}$ Genauso wie es auch damals ein Kampf war, in dem eine affektive Verbundenheit mit denjenigen, deren Leben bedroht war, eine wichtige Rolle spielte (»a wave of dying friends«)..$^{5}$ In Bezug auf das Ja der interviewten Person zum intersektionalen LesMigraS-Wir ist ein gemeinschaftsbildender Aspekt relevant, der nicht explizit in dieser Art und Weise in den diskursiven Verhandlungen der Einrichtung thematisiert wird, der aber an die bewegungsgeschichtliche Bedeutung von "queer« anknüpft. ${ }^{6}$

In den Villa-Diskursen spielt vor allem im Zusammenhang mit dem Projekt »Queer Base« die Auseinandersetzung mit Rassismus und Mehrfachdiskriminierung eine zentrale Rolle. Schon früh haben die Villa-Aktivist"innen im Namen einer »intersektionalen Solidarität «Abschiebe-Praktiken kritisiert, und mit "Queer Base« haben sie ein Projekt ins Leben gerufen, das Wohnraum für LGBITQ-Geflüchtete organisiert. Ähnlich wie die Person von LesMigraS versteht auch der interviewte Villa-Aktivist intersektionale queere Politiken als einen existenziellen politischen Kampf. Dabei knüpft die interviewte Person explizit an die Diskurse zur Aids-Krise an: 
»Also ich glaube, >queer bedeutet Aktivismus und Auflehnung gegen ein tödliches, prekäres System, das uns umbringt in der einen oder anderen Form. Und ich glaub auch, wenn jetzt Aids nicht mehr das große Thema ist, wir finden die Konflikte und den tödlichen neoliberalen Scheißdreck überall, ob das, keine Ahnung, Polizeigewalt in den USA gegen Schwarze ist, ob das, äh, keine Ahnung, die steigenden Mietpreise sind, die die jungen Menschen aus den Städten verdrängen. Ich glaub, das sind die Punkte, wo man ansetzen kann. Ja, das ist alles >queer «.«

Diese Bedeutung, die aus der Perspektive der ersten Person einem intersektionalen Wir zugeschrieben wird, knüpft in mehrfacher Hinsicht an die Villa-Diskurse an. Prekarität - ein Begriff, der die ungleiche Verteilung von Gefährdetheit beschreibt - ist in den Villa-Diskursen explizit der gemeinsame Nenner, um den sich das intersektionale VillaWir anordnet. Ähnlich wie der interviewte Aktivist stellt auch die Villa - ikonographisch mit den »blutenden Händen« auf dem Banner »Abartige gegen Abschiebung« - eine Verknüpfung zwischen den Toten der Aids-Krise und den Toten im Mittelmeer, den neuen death-figures, her. Ebenso wird, wie in den Villa-Diskursen üblich, auch aus der Perspektive des Aktivisten explizit der Bezug zur Villa-Geschichte hergestellt, wenn als Beispiel für die neue Prekarität Wohnverhältnisse angeführt werden. Der Villa-Aktivist* sagt ganz klar Ja zu der Art und Weise, wie sich das Villa-Wir in der Thematisierung von Mehrfachdiskriminierung diskursiv hergestellt.

Allerdings lehnt die Person aus der Villa nach vielen Jahren Aktivismus eine bestimmte Form, die das intersektionale Villa-Wir angenommen hat, ab - eine intersektionale Gemeinschaft, in der die selbstkritische Auseinandersetzung mit Rassismus als identitätspolitisch erlebt wird:

»Was super ist, was ur toll ist: das Haus hat über dreißig Jahren weißen Cis-Männern und -Frauen gehört und jetzt ist eine neue Generation da ... Aber ich bin kein Freund dieser Form der Identitätspolitik, wenn sie dogmatisch wird. Ich glaub, das hat man so ein bisschen gemerkt. Wie gesagt, ich war geduldet. Und es hat auch keinen Spaß gemacht. Ich hab es so ein Jahr durchgebissen und dann hab ich mir gedacht: na!«

Ähnlich wie das queere Wir wird auch ein intersektionales Wir verneint, wenn es identitätspolitisch ist. Inwiefern wird das intersektionale Wir aber als identitätspolitisch bezeichnet? Was macht die intersektionale Gemeinschaft aus der Perspektive des Akteurs* zu einer identitätspolitischen? Ein intersektionales Wir wird als identitätspolitisch erlebt, wenn die Frage, wer Teil der Gemeinschaft ist - in dem Fall: wer in den Villa-WGs wohnt -, an die ethnische Zugehörigkeit - race - der Personen geknüpft wird:»Jede neue Person, die einzieht, wurde zuallererst nach dem race-Kriterium beurteilt. Völlig irrelevant, ob die Person mitarbeiten wollte oder nicht, ob sie mitarbeiten konnte oder nicht. Es wurden Leute raus, es wurden Leute gegangen." Eine intersektionale Gemeinschaft wird auch als identitätspolitisch erlebt, wenn die selbstkritische Auseinandersetzung mit Rassismus moralisiert und individualisiert wird. Ein Aktivist* spricht von einer internen Debattenkultur, in der »weiße« Aktivist*innen ständig mit dem Vorwurf konfrontiert waren, sie seien rassistisch (Diese Vorwürfe kamen mitunter von Aktivist*innen die selbst »weiß« sind): 
»Die Leute, die Weißen, waren immer in irgendeiner Form in einen Konflikt involviert, der als rassistisch gelesen wurde oder der rassistisch war. Die Leute haben sich nichts mehr getraut zu sagen. So. Und ich ja genauso. Ich hab irgendwie überhaupt keine Kraft mehr gehabt, da jetzt irgendwie zu diskutieren, warum die Person nichts für die Villa ist. [...] Uns wurde um die Ohren gehauen, also uns weißen Rassisten von Weißen, dass wir so billig wohnen, dass das eine Frechheit ist. Es war so arg. Da hab ich mir das erste Mal so gedacht, so woa, was passiert da eigentlich."

Das intersektionale Villa-Wir, das insofern als identitätspolitisches erkannt wird, als die ethnische Zugehörigkeit - race - bestimmt, wer Teil der Gemeinschaft ist und wer nicht, und insofern, als die selbstkritische Auseinandersetzung mit Rassismus individualisiert und moralisiert wird, wird aus der Perspektive des Aktivisten abgelehnt, weil es ein affektives Unbehagen auslöst, das einem Gemeinschaftsgefühl entgegensteht: »Ich kann mich an ein Plenum erinnern, ich bin noch nie gesessen mit so vielen Leuten, die ich kenne, die ich gut kenne, und hab so ein beklemmendes Gefühl gespürt, das war so schmerzhaft, und ich war noch nicht einmal involviert in den ganzen Konflikt.«

Aus der Perspektive der Aktivist*innen wird jeweils ein intersektionales Wir, das einen gemeinsamen existenziellen Kampf begründet, angenommen. Ähnlich wie in Bezug auf den Begriff »queer«, ist auch hier das Identitätspolitische das, was abgelehnt wird. Aus subjektiver Perspektive spielen besonders persönlich-affektive Aspekte eine wichtige Rolle. In dem einen Fall ist die affektive Verbundenheit mit denen, die einem nahe sind, ein wichtiger Grund für das intersektionale Engagement. Im anderen Fall ist es ein affektives Unbehagen, das durch eine bestimmte Form, die das intersektionale Wir angenommen hat, ausgelöst wird, das einem »Gemeinschaftsgefühl« entgegensteht und zu einer Beendigung des Aktivismus führt.

\section{Pride und Coming-out}

Auch auf die Pride und das Coming-out wird in den Interviews in einem affirmativen Sinne Bezug genommen. Die Milchjugend-Aktivist*innen bejahen bestimmte Aspekte des im Sprechen über die Pride und das Coming-out diskursiv hergestellten kollektiven Wir. Die Pride wird erstens als Raum gemeinsam geteilter Euphorie bejaht, zweitens als Raum des stolzen und selbstbewussten Beisammenseins. Das Interview mit den beiden Milchjugend-Aktivist*innen hat am Festival-Gelände auf der Zürich-Pride 2017 stattgefunden. Auf die Frage, wie sich die Aktivist*innen eine perfekte queere Welt vorstellen, verweisen sie auf die Pride und das, was sie gerade hier auf der Pride geschaffen haben: »So [lacht]! Und niemand muss arbeiten [lacht]. Ja, junge Menschen, die sich ausprobieren, das ist für mich das größte Glück. Ohne Schuldgefühle, aber mit viel Mut.« Auch aus der Perspektive der Aktivist*innen wird das diskursiv hergestellte euphorische Ja zur Pride angenommen. Für die interviewten Personen, die auf die Frage nach einer perfekten queeren Welt, wohl auch von der aktuellen Pride-Euphorie getragen, antworten: »So!«, materialisiert sich auf der Pride, wofür sie sich engagieren. Es ist aber nicht nur ein in der Euphorie begründetes Ja. Der Grund, warum die Aktivist*innen ein kollektives Wir, das gemeinsam auf der Pride erscheint, annehmen, ist die Möglichkeit, dort einen Raum zu schaffen, an dem Jugendliche sich mutig und ohne Schuldgefühle ausprobieren können. Damit knüpfen die Aktivist*innen an die Gemeinschaft an, die 
in der projekteigenen Öffentlichkeit über fast alle verhandelten Themen hinweg hergestellt wird: ein Wir, das sich gegenseitig auf dem Weg, zu einem selbstbestimmten und selbstbewussten Subjekt zu werden, bestärkt und unterstützt. Diese subjektive Begründungsebene der Aktivist*innen schließt auch an den Coming-out-Diskurs der Milchjugend an. Auch dort konstituiert sich die Einrichtung als eine Gemeinschaft, die im Sprechen über das Coming-out die Möglichkeit eröffnet, zu einem stolzen und handlungsmächtigen Subjekt zu werden. Während von den Aktivist"innen die im Coming-outDiskurs der Milchjugend implizit bejahte Schuld abgelehnt wird - »[o]hne Schuldgefühle, aber mit viel Mut« - , werden die einseitigen Happy-End-Geschichten des Comingout-Diskurses der Milchjugend auch von den Aktivist*innen bejaht: »Negativität haben wir nie zugelassen. Also so, so irgendwie Artikel, die so, wie schlimm alles ist, und ich bin traurig und ich bin depressiv, und es musste immer auch einen Dreh haben noch, so von dieser Selbstermächtigung, das war eigentlich das Einzige, das wir immer//das habe ich immer geblockt." Das kollektive Wir der Milchjugend, das in den Verhandlungen der Pride und des Coming-out hergestellt wird, ist auch für die Aktivist"innen von Bedeutungen. Teil einer Gemeinschaft sein zu können, die Räume herstellt, die Jugendlichen die Möglichkeiten eröffnen, sich als stolzes und handlungsmächtiges Subjekt zu konstituieren, ist ein zentraler Grund, sich für die Milchjugend zu engagieren. Genauso wie die geteilte Euphorie als affektiver gemeinschaftsbildender Affekt von Bedeutung ist.

Auch die interviewte Lambda-Aktivist*in berichtet, dass sich auf dem CSD zeige, wofür sie* sich einsetzt. Jugendliche kommen zum CSD-Wagen von Lambda und erzählen, dass sie sich im Anschluss an die Sensibilisierungs- und Empowerment-Workshops des »queer@school«-Projektes geoutet hätten: »Lambda hat ja einen CSD-Wagen jedes Jahr, und was ich immer sehr schön fand an den CSD-Wägen, war, dass Schüler*innen vorbeikamen, die in unseren Workshops waren, und sie sich dann irgendwie als "queer « geoutet haben und dann uns besucht haben an unserem Wagen und uns dann erzählt haben."Der CSD ist also der Ort, an dem sichtbar wird, was die Aktivist* in erreicht hat: Jugendliche, die sich geoutet haben. Das Coming-out von Jugendlichen als eine subjektiv relevante Bedeutung, zu der die interviewte Aktivist*in Ja sagt, findet sich auch in den diskursiven Verhandlungen von Lambda wieder. In der »Out! « konstituiert sich Lambda explizit als Austausch- und Unterstützungsraum, der grundlegend um das erfolgreiche Coming-out angeordnet ist. Daran anknüpfend ist das Lambda-Wir, das die Möglichkeiten für ein erfolgreiches Coming-out eröffnet, aus der Perspektive der Aktivist*in bedeutungsvoll.

Im Fall von TransInterQueer e.V. nimmt die interviewte Person wenig Bezug auf die konkreten diskursiven Aushandlungsprozesse, die bei TrIQ im Analysezeitraum aktuell sind. Das kann erstens daran liegen, dass die interviewte Person zur Zeit des Interviews erst seit kurzem bei TrIQ aktiv war, zweitens daran, dass es die Aufgabe der interviewten Person war, statt der Lobbyarbeit, die bisher eine zentrale Rolle gespielt hatte, vermehrt Austauschräume zu schaffen. ${ }^{7}$ Die Person von TrIQ sagt vor allem Ja zu den Möglichkeiten des Austauschs, die bei TrIQ geschaffen werden sollen: »Also ich war schon ganz oft dabei, wenn Inter* also jetzt nicht unbedingt nur im Kontext von TrIQ 
sondern generell, wenn so Leute sich zum ersten Mal im Community-Kontext befinden und das halt irgendwie so ganz super emotionale Momente sind. Und ich glaub, das sind so für mich die großartigsten oder waren die großartigsten Sachen, so.« Aus der Perspektive der interviewten Person wird insofern ein Aspekt des diskursiven TrIQ-Wir als relevant erachtet, als TrIQ als Austauschraum bejaht wird. Ähnlich wie bei der Villa und LesMigraS wird auch hier die aus subjektiver Perspektive relevante affektive Komponente betont. Es ist das affektiv Bewegende - »so ganz super emotionale Momente«-, für das es sich zu engagieren lohnt.

\subsection{Strukturelle Anforderungen und das eigene gute Leben}

Nachdem die Art und Weise, wie die Aktivist"innen sich zu dem "Queer« und den diskursiven Bedeutungsanordnungen ihrer Projekte ins Verhältnis setzen, in den Blick genommen wurde, soll es nun darum gehen, welche weiteren Bedingungs-BedeutungsBegründungs-Konstellationen aus der Perspektive der Akteur*innen von Gewicht sind.

\section{Materielle und strukturelle Bedingungen}

Die interviewten Personen thematisieren alle auch die Bedingungsebene. Zum einen sprechen sie von einer materiellen Notwendigkeit der Existenzsicherung, die mit dem Aktivismus mitunter schwer vereinbar sei, zum anderen von strukturellen Anforderungen des Aktivismus, die teilweise in einem Widerspruch zu subjektiven Bedürfnissen stehen würden. Ersteres verweist vor allem auf die Bedeutung, die der Aktivismus für die interviewten Personen hat. Denn diese entscheiden sich mitunter trotz damit einhergehender finanzieller Schwierigkeiten für den Aktivismus: »Arbeiten, Uni und Aktivismus wär sich nicht ausgegangen. [...] Als das mit dem Studium fertig war, bin ich dann erst mal mit einem Minus irgendwie dagestanden und hab dann auch einfach dringend einen Job gebraucht, und da hab ich gemerkt, okay, Job und Aktivismus, das ist mir zu mühsam.«(IP3) Auch andere Aktivist*innen berichten davon, finanzielle Abstriche in Kauf zu nehmen, und sich stattdessen für den der eigenen politischen Haltung entsprechenden Aktivismus zu entscheiden. Für diejenigen, für die der Zugang zum Arbeitsmarkt begrenzt ist - das sind im Fall meiner Interviewpartner*innen zumeist Trans* - und Inter*-Personen -, eröffnet der Aktivismus aber auch die Chance auf einen Arbeitsplatz, an dem sie erscheinen und sein können, wie sie wollen. Eine Person berichtet davon, vorher in einem ähnlichen Projekt in einer anderen Stadt aktiv gewesen zu sein und wegen der Möglichkeit der Bezahlung die Stadt und damit auch das Projekt gewechselt zu haben:

»Also in [...] [Stadt] hatte ich keinen Job, und gleichzeitig hab ich da ganz viel bei [...] [queeres Projekt] gearbeitet. Ich hab ganz ähnlich da gearbeitet. Und genau, wir hatten aber nie Förderungen in Höhen, dass wir uns selber dafür hätten bezahlen können in den allermeisten Fällen. Und drum hat sich das für mich super angeboten, das als bezahlte Stelle, also Teilzeitstelle zu haben.«(IP 5)

Die Aktivist*innen - und das gilt nicht nur für den queeren Aktivismus - müssen sich mitunter zwischen einer queeren politischen Haltung und den Möglichkeiten der finan- 
ziellen Existenzsicherung entscheiden. Die Entscheidung für den queeren Aktivismus verweist in diesen Fällen auf einen hohen Grad der Identifizierung mit dem queeren kollektiven Wir. Eine Ausnahme sind vor allem Trans*- und Inter*-Personen, die jenseits des Aktivismus wenig Chancen auf einen Arbeitsplatz haben, an dem sie erscheinen können, wie sie wollen. Für sie ist der queere Aktivismus eine Option, beides politische Haltung und materielle Existenzsicherung - zu vereinen.

Auf der Bedingungsebene werden zweitens aber auch strukturelle Anforderungen des politischen Aktivismus benannt, die in einem Widerspruch stehen mit den Bedürfnissen der Aktivist*innen, die das, was das queere Wir ihnen verspricht, angenommen haben. Es werden vier solche strukturellen Bedingungen des Aktivismus genannt: erstens die Vereinzelung in der aktivistischen Arbeit, zweitens historisch gewachsene Strukturen, drittens strategische Erfordernisse und viertens eine offene Projektstruktur.

Im Gegensatz zu einer queeren Bedeutungsanordnung, die das Verbindende der eigenen Gemeinschaft zum Ausgangspunkt gemeinsamer Politiken macht, wird erstens die Erfahrung der Vereinzelung in der aktivistischen Arbeit beschrieben: »Auch so im Haus selbst, die Sichtbarkeit und so das kollektive Gefühl zu sagen, shey wir Queerdos, wie wir durch die Straßen ziehen und Nazis verprügeln<, das habe ich nie gehabt, das hat es nie gegeben. Das war immer so ich, der arbeitet, ich, der neoliberal vereinzelte Mensch, der seine Arbeitskraft in das Projekt seines Herzens einbringt.« (IP3) Die strukturelle Bedingung des Aktivismus, der vereinzelte Arbeit erfordert, steht in einem Widerspruch zu einem queeren Anspruch, ein kollektives Wir jenseits von neoliberaler Vereinzelung herzustellen. Ein queeres Wir, das entgegen einer neoliberalen Vereinzelung die Verbundenheit und wechselseitige Abhängigkeit betont, ${ }^{8}$ kann aus der Perspektive der Aktivist*innen nicht angenommen werden, weil die real stattfindende vereinzelte Verausgabung der Arbeitskraft dem entgegensteht. Sie verunmöglicht das Aufkommen kollektiver Affekte der Verbundenheit, die eine solche Gemeinschaft ermöglichen würden. Die Aktivist*innen fühlen sich alleine, nicht verbunden. Die Bedingungsebene - die reale Arbeit, die im Aktivismus oft vereinzelt geleistet werden muss - wird als Widerspruch zu den Bedeutungsanordnungen, die auch aus der Perspektive der ersten Person relevant sind - ein kollektives Gefühl der Gemeinschaftlichkeit - , hervorgehoben.

Es wird, zweitens, auch von einem Widerspruch zwischen dem persönlichen Bedürfnis nach einem offenen Aktivismus, das wiederum in den queeren Bedeutungsanordnungen der Projekte angelegt ist, und den erlebten historisch gewachsenen Strukturen berichtet:

»Ich bin da ein bisschen in Strukturen geraten, die es wie gesagt schon seit $\mathrm{x}$ Jahren gegeben hat, die einerseits sehr offen waren, aber auch andererseits total zu. Es hat viele Leute gegeben, die schon lange dabei waren, die gesagt haben, das war immer schon so, das machen wir so. Das war im Endeffekt ein bisschen das Gegenteil von dem, was ich mir erwartet hab. So mich befreien. Das war sehr einengend und auch nicht sehr lustig und es war sehr mühsam, es war sehr harte Arbeit, um das sehr knapp zusammenzufassen.«(IP3) 
Auch die politisch-strategischen Erfordernisse, Netzwerke und Ressourcen zu nutzen, geraten, drittens, in einen Konflikt mit dem persönlichen Bedürfnis von Seiten der Akteur*innen, $\mathrm{zu}$ dem selbstermächtigten Subjekt zu werden, das eine queere Bedeutungsanordnung ihnen eröffnet:

»[G]erade so in der politischen Lobbyarbeit find ich es halt oft super schwierig, mich so ein bisschen zusammenzureißen unter Anführungszeichen, weil ich so das Gefühl habe, wenn ich so Teil von so Diskussionsrunden oder so bin, dass es dann ganz oft passiert, dass ich oder andere schon krass krass diskriminiert werden [...] und man dann aber trotzdem strategisch sein muss irgendwie, um bestimmte Connections aufrechtzuerhalten, um irgendwie bestimmte Zugänge zu Ressourcen oder weiteren Diskussionen zu haben.«(IP5)

Beide Male entsprechen die persönlichen Bedürfnisse den diskursiv hergestellten Zielen ihrer Projekte. Diese konstituieren sich als ein queeres Wir, das offen sein will und Menschen ermächtigt, sich gegen die erfahrenen Diskriminierungen zu wehren. Die Aktivist"innen haben dieses Wir angenommen, stoßen aber aufgrund struktureller Anforderungen des Aktivismus auf Widersprüche. Sie erfahren ihre Gemeinschaft nicht als offen - historisch gewachsene Strukturen und politisch-strategische Interessen verunmöglichen mitunter diese Offenheit.

Umgekehrt ist es aber, viertens, auch eine offene Projektstruktur - die es in einem queeren Sinne ermöglicht, immer wieder die Frage zu stellen, »wer dieses Wir ist und sein will« -, die mit dem persönlichen Bedürfnis nach politischer Handlungsmächtigkeit kollidiert: »Die Hauptkonfliktlinien waren immer die gleichen, und zwar: welche Politik machen wir, was sind wir für ein Haus, wer sind wir, wie arbeiten wir? Und dadurch gab es viel Raum für Diskussion, aber wenig Raum für Praxis. Wir sind so ein bisschen an den Diskussionsstrukturen im Endeffekt gescheitert.« (IP3) Auf der einen Seite versprechen die queeren Bedeutungsanordnungen Handlungsmächtigkeit beziehungsweise ist es das, was die Aktivist*innen dort suchen. Auf der anderen Seite verunmöglicht es eine offene Projektstruktur, die im Sinne eines queeren Wir ist, sich als handlungsmächtiges Subjekt zu konstituieren.

Die Aktivist*innen sind mit dem Widerspruch konfrontiert, auf der einen Seite zu den Bedeutungsanordnungen der queeren Projekte - Offenheit, Verbundenheit und die Möglichkeit, zu handlungsmächtigen, widerständigen Subjekten zu werden - Ja zu sagen, und erfahren auf der anderen Seite, dass aufgrund struktureller Bedingungen diese diskursiven Bedeutungsanordnungen selbst nicht ganz aufgehen. Die queeren Projekte werden aufgrund historisch gewachsener Strukturen als geschlossen erlebt, Diskriminierung muss im Namen strategischer Interessen ausgehalten werden und die für ein offenes »Queer« zentrale Diskussion darüber, »wer das Wir sein will«, führt dazu, dass sich nichts bewegt und die Aktivist*innen sich als wenig handlungsmächtig erfahren.

\section{Das eigene gute Leben}

Die Akteur*innen nehmen bestimmte Bedeutungsanordnungen des queeren Aktivismus an: ein "Queer « als Haltung oder Handlung, ein intersektionales Wir, ein Wir, das die Möglichkeit eröffnet, stolz und selbstbewusst zu sein und sich zu outen, oder ein Wir, das Austauschräume eröffnet. Gleichzeitig verunmöglichen es bestimmte struk- 
turelle Bedingungen der aktivistischen Praxis, dass die von den Akteur*innen angenommenen queeren Bedeutungsanordnungen aufgehen. Warum entscheiden sich die Personen mit Blick auf diese Widersprüche für oder gegen den queeren Aktivismus? Es wurden bereits einige Gründe genannt. Für ein queeres Wir sprechen affektive Verbundenheit und existenzielle Notwendigkeit sowie ein Mehrals Identität. Gegen ein queeres Wir sprechen Ein- und Ausschlüsse sowie die Verunmöglichung eines Gemeinschaftsgefühls. Welche anderen Gründe haben die Aktivist*innen, sich für oder gegen die queere Gemeinschaft ihrer Einrichtungen zu entscheiden?

Für die Aktivist*innen spielt auch das eigene Leben ${ }^{9}$ in der Entscheidung für oder gegen den Aktivismus eine Rolle. Sie berichten davon, dass sie ihrer von der Norm abweichenden vergeschlechtlichten und/oder sexuellen Zugehörigkeit nicht entkommen können. Der Aktivismus stellt für sie eine Möglichkeit dar, sich die Auseinandersetzungen in Bezug auf diesen Teil des von der Norm abweichenden eigenen Lebens aussuchen zu können:

»Also was ich mir nicht aussuchen kann, ist, dass ich eine Auseinandersetzung mit Inter ${ }^{*}-$ Themen in meinem Leben habe. So. Das ist so, ob ich es haben will oder nicht. So. Und ich glaub, es ist auch ein bisschen selfcare in die Richtung - okay, aber ich kann mir zumindest aussuchen oder zu einem Teil zumindest auch aussuchen, welche Auseinandersetzung das ist.«(IP5)

Aus der Perspektive der Akteur*innen ist der Aktivismus eine Möglichkeit, das eigene Leben nicht derart von Geschlechternormen regieren zu lassen. Der Aktivismus bietet den Akteur*innen die Möglichkeit, bis zu einem gewissen Grad zu einem handlungsmächtigen Subjekt zu werden. Dabei geht es den Aktivist*innen nicht nur darum, für sich selbst Möglichkeiten zu finden, sich nicht derart regieren zu lassen, sondern sie wollen generell etwas gegen Ungleichheitsverhältnisse unternehmen. Auch hier wird in zweifacher Hinsicht der Bezug zum eigenen Leben betont. Zum einen insofern, als die eigene Abweichung von den Geschlechternormen den Aktivist*innen Ungleichheiten als subjektiv erkennbare und relevante Bedeutungsebene eröffnet hat: "Also äh, ich erlebe bewusst Ungerechtigkeiten und Dinge, die ich als falsch empfinde, Gesellschaft ohne Akzeptanz, mit Menschen, die ich sehe, denen Möglichkeiten fehlen. Und ich kann mit meinem Aktivismus etwas dagegen tun. Das fühlt sich sehr gut an.«(IP2) Zum anderen - und das klingt in »das fühlt sich sehr gut an« bereits an - insofern, als sie im Aktivismus die Antwort auf die Frage danach, wie sie ein "gutes Leben« führen können, suchen:

»Es ist natürlich auch die Erfahrung von purpose, den man findet. Von Zweck, dahingehend, dass man sich irgendwie mit etwas verbunden fühlt, dass man etwas hat, wofür man gerne arbeitet. [...] Das finde ich eine großartige Ressource, weil es mir sehr viele Entscheidungen abnimmt, weil es mir sehr viel Suchen abnimmt. [...] So in meinem Fall einen recht offensichtlichen positiven Zweck, dass ich einen positiven Einfluss in meinem Lebe haben kann.«(IP4)

9 Das eigene Leben wird in der vorliegenden Untersuchung im Sinne Butlers als immer schon »enteignetes verstanden. 
In der Entscheidung für oder gegen den Aktivismus spielen nicht nur die Möglichkeiten eines queeren Aktivismus, jenseits einfacher ein- und ausschließender Identitätspolitiken zu agieren, nicht nur die affektive Verbundenheit mit denjenigen, die prekär leben, und nicht nur die Möglichkeit, im Aktivismus ein Gemeinschaftsgefühl zu erfahren, eine Rolle, sondern auch die Möglichkeit, das eigene Leben handlungsmächtig und "gut« führen zu können.

\subsection{Affekte als gemeinschaftsbildendes Moment}

Aus der Perspektive der Subjekte sind verschiedene Bedingungs- und Bedeutungskonstellationen relevant, die teilweise für den Aktivismus generell gültig sind, meistens aber auf eine queer-spezifische Thematik abzielen. Zunächst einmal zeigt sich, dass die eigene Identität - die individuelle Selbstverortung - keine Rolle spielt in Bezug auf die Art und Weise, wie die Akteur*innen sich in ein Verhältnis zu dem kollektiven Wir ihrer Projekte setzen. Die Aktivist"innen identifizieren sich völlig unabhängig davon, ob sie das queere Wir ihrer Gemeinschaft annehmen oder ablehnen, selbst als "queer« oder eben »nicht queer«. »Queer« als Band, das die einzelnen Projekte zusammenhält, muss aus der Perspektive der Akteur*innen keine Identitätsbezeichnung sein, mit der sich die Aktivist*innen als das, was sie selbst sind, identifizieren können. Im Gegenteil, die Aktivist"innen identifizieren sich mit queer als Haltung oder Handlung, nicht als Kategorie, die bezeichnet, »wer sie sind«. Sie wollen eine Gemeinschaft, die insofern "queer « ist, als sie gerade nicht identitätspolitisch ist. Die subjektiven Bedeutungen knüpfen an die Bedeutungen von "queer«, die queer-theoretisch und bewegungsgeschichtlich hergestellt wurden, an. Die Aktivist"innen wollen ein "Queer«, das Bündnisse jenseits von Identitäten möglich macht, das subversiv ist, und ein Wir, aus dem keine Gruppen per se ausgeschlossen sind. Auch wenn es nicht unbedingt mit dem Namen »queer« bezeichnet wird, knüpfen die Aktivist*innen auch an ein bewegungsgeschichtliches "Queer an, das als gemeinsamer Kampf gegen den Tod verstanden wird. Wenn sie auf der Bedeutungsebene ein »Queer« erkennen, das entsprechend der aufgezählten Bedeutungen ein Jenseits von Identitätspolitiken verspricht, nehmen sie das kollektive queere Wir eher an, als wenn es ihnen zu ausschließend erscheint.

Aus der Perspektive der Akteur*innen werden größtenteils auch die in den diskursiven Aushandlungen der projektinternen Öffentlichkeiten zentralen Aspekte bejaht. Sie wollen ein queeres Wir, das intersektional ist, ein queeres Wir, das stolz ist und das Menschen die Möglichkeiten zum Coming-out eröffnet. Insofern, als die Aktivist*innen die in den Diskursen der Projekte hergestellten, aber auch die theoretischen wie bewegungsgeschichtlichen Bedeutungen von »queer « annehmen, findet eine Identifizierung mit den Bedeutungen des Begriffs statt. Diese Identifizierung äußert sich auch, wenn die Aktivist"innen davon berichten, dass die Widersprüche zwischen den verinnerlichten Bedürfnissen nach einem offenen Wir, aber auch einem Wir, das dazu ermutigt, zu einem handlungsmächtigen und sich gegen Diskriminierung wehrenden Subjekt zu werden, und den realen strukturellen Bedingungen politischer Praxis schwer auszuhalten sind. Gleichzeitig ist die Identifizierung mit bestimmen Bedeutungen von "queer» bis zu dem Grad gegeben, in dem ökonomische Kosten für das queere Wir in Kauf ge- 
nommen werden. Es scheint also in dem Sinne eine Identifizierung mit »queer« zu geben, als die subjektiven Bedeutungen mit den queer-theoretischen, aktivistischen und bewegungsgeschichtlichen Bedeutungen übereinstimmen. Diese Identifizierung ist für die Akteur*innen aber vor allem dann bedeutungsvoll, wenn der queere Aktivismus sie auf eine bestimmte Art und Weise zu affizieren vermag.

Es werden drei affektive Bezüge genannt, die aus der Perspektive der Aktivist"innen eine entscheidende Rolle bei der Entscheidung für oder gegen das queere Wir spielen: erstens eine affektive Verbundenheit mit denen, die existenziell bedroht sind, zweitens eine affektive Verbundenheit mit der eigenen Gemeinschaft und drittens das Gefühl, etwas »Richtiges « zu tun. Ein Grund, sich für die queere Gemeinschaft zu entscheiden, ist die affektive Verbundenheit mit denen, die existenziell bedroht sind. Die existenzielle Bedrohung von Menschen, die einem nahe und wichtig sind, mitzuerleben, vermag aus der Perspektive des Subjekts ein wichtiger Grund zu sein, sich für einen queeren Aktivismus zu entscheiden, der genau bei dieser alltäglich erlebten existenziellen Bedrohung ansetzt und gegen sie arbeitet. Es ist für die Aktivist*innen aber auch von Bedeutung, dass die eigene queere Gemeinschaft ein Gefühl der Verbundenheit hervorzubringen vermag. Das äußert sich in dem Ja der Aktivist*innen zu einer gemeinsamen »Euphorie« auf der Pride, die genau eben jene Verbundenheit ausdrückt. Das Gemeinschaftsgefühl wird aber vor allem auch dann als ein Grund für und gegen den queeren Aktivismus betont, wenn es fehlt. Beispielsweise dann, wenn die strukturellen Bedingungen vereinzelter Arbeit ein solches affektives Gemeinschaftsgefühl verunmöglichen. Das Gemeinschaftsgefühl kann auch nicht aufrechterhalten werden, wenn interne Debattenkulturen ein affektives Unbehagen auslösen. Gleichzeitig suchen die Aktivist*innen selbst im Aktivismus ein "gutes Gefühl«, die Antwort auf die Frage nach dem »richtigen Leben«. Die Möglichkeit, im queeren Aktivismus etwas gegen vergeschlechtlichte Ungleichheiten zu unternehmen, gibt ihnen das Gefühl, dass das eigene Leben gut und sinnvoll ist.

Diese affektiven Bezüge, die es aus der Perspektive der Aktivist"innen braucht, verweisen in mehrfacher Hinsicht auf Judith Butler, die das Politische in einer konstitutiven Abhängigkeit voneinander - und damit jenseits einfacher identitätspolitischer Ein- und Ausschlüsse - zu begründen versucht. Inwiefern fügen sich die affektiven Gemeinschaftsbezüge, die aus der Perspektive der Aktivist*innen relevant sind, in eine solche Idee des Politischen ein? Und wo verweist beides - eine solche Idee des Politischen und die subjektiven Handlungsgründe - womöglich auf die Grenzen des jeweils anderen? Judith Butler betont die Notwendigkeit einer politischen Gemeinschaft, die sich auf dem gründet, was die Menschen verbindet. Die konstitutive Abhängigkeit der Menschen voneinander macht sie zugleich verletzlich und bindet sie ethisch aneinander. ${ }^{10}$ Eine affektive Verbundenheit mit denjenigen, die existenziell bedroht sind, schließt gleich in zweifacher Hinsicht an Butlers Idee einer ethischen Verantwortung an. Zum einen insofern, als die Verbundenheit betont wird, zum anderen insofern, als die existentielle Bedrohung, die ja gerade auf unsere Verletzlichkeit zurückgeht und abhängig ist von einem bestimmten gesellschaftspolitischen System, zentral ist. Anders als in der Perspektive der interviewten Akteur*innen, in der eine Verbundenheit mit denjenigen, die 
einem nahe sind, als bedeutungsvoll hervorgehoben wird, umfasst Butlers ethische Verantwortung jedoch darüber hinausgehend auch diejenigen, mit denen wir ungewählt zusammenleben. ${ }^{11}$

Auch das Thematisieren des Gefühls der Verbundenheit in der eigenen politischen Gemeinschaft, das aufgrund neoliberal vereinzelter Arbeit nicht aufkommt, schließt an Judith Butlers Überlegungen des Politischen an. Ihr zufolge soll es gerade die aus der wechselseitigen Abhängigkeit resultierende Verbundenheit sein, die gegen neoliberale Vereinzelungen und Vorstellungen von Autonomie ein politisches Subjekt begründet. ${ }^{12}$ Aus der Perspektive der Aktivist"innen verhindert die vereinzelte Arbeit einen solchen Gemeinschaftsbezug. Es scheint eine Herausforderung zu sein, eingelassen in neoliberale Strukturen der Vereinzelung, ein gegen diese Strukturen gewendetes Gefühl der Verbundenheit herzustellen.

Neben dem affektiven Bedürfnis nach gemeinschaftlicher Verbundenheit wird auch das moralische Gefühl als zentraler Grund für den Aktivismus genannt. Während die Moral aus der Perspektive des Subjekts einerseits keine affektive Atmosphäre erzeugen darf, in der ein gemeinschaftliches Gefühl der Verbundenheit verunmöglicht wird, so suchen die Akteur"innen doch für sich selbst im Aktivismus eine Antwort auf die Frage nach dem »richtigen Leben «. Aus der Perspektive des Subjekts scheint es von Bedeutung zu sein, dass die Akteur*innen als das, was sie durch den Aktivismus geworden sind und ständig werden, auf der (moralisch) »richtigen « Seite stehen. Doch ist das nicht das Gegenteil von jener affektiven Verbundenheit, die von den Aktivist*innen sonst eingefordert wird? Handelt es sich bei dem Bedürfnis nach einem "guten Gefühl« nicht gerade um »die narzisstische Selbstbefriedigung, sich für besser halten zu dürfen, als die anderen sind «? ${ }^{13}$ Steht nicht ein individuelles Bedürfnis nach einem »richtigen Leben « durch den Aktivismus einer queeren aktivistischen Haltung entgegen, der zufolge der Aktivismus niemals festschreiben kann, wie dieses »richtige Leben« auszusehen hat? Judith Butlers Überlegungen zufolge müssen beide Fragen verneint werden. Sie versteht das moralische Gefühl nicht in erster Linie als Ausdruck eines Ichs, das sich von anderen als etwas Besseres abgrenzen will, sondern als einen Ausdruck der Selbsterhaltung, der nicht in einer Abgrenzung von den anderen, sondern im Gegenteil in einer Abhängigkeit und Verbundenheit mit den anderen gründet: »Der andere ist Mittel meines eigenen Überlebens, und Schuldgefühle, ja die Moral selbst, entstehen schlicht aus diesem Verlangen der Selbsterhaltung, das vor allem durch meine eigene Destruktivität bedroht ist. [...] Schuldig bin ich, weil ich versucht habe, ein Band zu zerstören, das ich für mein Überleben brauche. ${ }^{14}$ Wenn andere Leben zerstört werden, fühlen wir uns Butler zufolge schuldig, weil wir selbst aufgrund unserer »ergebnisoffenen Sozialität« konstitutiv verbunden sind mit diesen Leben. Ähnlich, wie im Fall der anderen beiden von den Aktivist*innen betonten affektiven Gemeinschaftsbezüge, scheint auch das moralische Gefühl auf eine konstitutive Abhängigkeit voneinander zu verweisen.

\footnotetext{
11 Ebd., S. 148.

12 Ebd., S. 192.

13 Sigmund Freud: Das Unbehagen in der Kultur, Frankfurt a.M.: Fischer Taschenbuch Verlag 2018, S. 106 .

14 J. Butler: Raster des Krieges, S. 50.
} 
Butler spricht sich auch dagegen aus, die Moral gänzlich aus der Politik zu verbannen. Sie plädiert gegen eine Politik, die auf Kosten der Zerstörung des Ichs die Moral aufgibt. Es braucht »ein >Ich<, das irgendwie in eine breitere soziale und politische Bewegung eintreten, mit ihr verhandeln und in ihr agieren muss ${ }^{15}$ Denn auch die politische Antwort auf die Frage nach dem »richtigen Leben« darf nicht in einer Zerstörung des Ichs und dessen Leben münden. ${ }^{16}$

Aus der Perspektive des Subjekts zeigt sich, dass die einzelnen Aktivist*innen sich mit den Bedeutungen von »queer« als Nicht-Identitäts-Kategorie identifizieren und queere Politiken jenseits von Identitäten wollen. Auch aus der Perspektive der ersten Person ist es nicht notwendig, ein politisches Wir um eine gemeinsam geteilte Identitäts-Kategorie anzuordnen. Es genügt, wenn sich die Aktivist*innen mit den Bedeutungen von »queer« identifizieren. Es ist allerdings aus der Perspektive der Akteur*innen für ein queeres Wir von Gewicht, auf verschiedenen Ebenen einen affektiven Gemeinschaftsbezug herstellen zu können.

15 J. Butler: Anmerkungen zu einer performativen Theorie der Versammlung, S. 272.

16 Ebd. 